\title{
You Do What in Your Microprobe?! The EPMA as a Multimode Platform for Nitride Semiconductor Characterization
}

\author{
Paul R. Edwards ${ }^{1}$, G. Naresh-Kumar ${ }^{1}$, Gunnar Kusch ${ }^{1}$, Jochen Bruckbauer ${ }^{1}$, Lucia Spasevski ${ }^{1}$, \\ Catherine G. Brasser ${ }^{1}$, Michael J. Wallace ${ }^{1}$, Carol Trager-Cowan ${ }^{1}$, and Robert W. Martin ${ }^{1}$ \\ ${ }^{1 .}$ Department of Physics, SUPA, University of Strathclyde, Glasgow, United Kingdom.
}

While the use of electron probe microanalysis (EPMA) is widespread in the geological and metallurgical sciences, it remains less prevalent in the field of semiconductor research. For these materials, trace element (i.e. dopant) levels typically lie near or beneath the detection limit of wavelength-dispersive Xray (WDX) spectrometers, while alloy compositions of ternary mixtures and multilayer structures can more readily be determined using X-ray diffraction techniques. The electron beam measurements more commonly applied to semiconductors remain transmission electron microscopy (for structural characterization), and scanning electron microscopy (topographic, optical and electrical information).

Despite this, there are many aspects of the EPMA that make it an attractive platform for all of these types of semiconductor characterization, particularly when combining compositional information from WDX with complementary and simultaneously-acquired signals. These advantages include: built-in light optics; a stable, quantified and high-current beam; and a combined large-area and high-resolution mapping capability. This allows the measurement of cathodoluminescence (CL), electron beam-induced current (EBIC) and electron channelling contrast imaging (ECCI) signals alongside WDX, which we apply to the investigation of visible and $\mathrm{UV} \mathrm{Al}_{\mathrm{x}} \mathrm{In}_{\mathrm{y}} \mathrm{Ga}_{1-\mathrm{x}-\mathrm{y}} \mathrm{N}$ materials, devices and nanostructures.

Excess charge carriers are fundamental to the function of semiconductor devices, so the ability to directly inject such carriers into a spatially localised region of a sample using an electron beam is invaluable. Optical information comes from the analysis of CL, the light emitted when the carriers recombine radiatively. By measuring in the hyperspectral imaging mode, we observe spatial variations in both emission intensity and wavelength, which correlate with compositional information (e.g. alloy fluctuations) and structural information (e.g. inhomogeneous strain). We extend this technique by looking at the effect on the emission of applying a bias across an electrically contacted sample (e.g. LED) [1]. This more closely matches the CL measurement conditions to those of a working device; at forward bias above the diode threshold this technique converges with electroluminescence (EL), and we now acquire EL hyperspectral images in the EPMA (with the beam off) for direct comparison with CL/WDX data acquired using the same scanning/optics setup. At below-threshold or reverse bias, the device junction can induce a current, resulting in an EBIC signal; as this depends on all recombination pathways, comparison with CL allows the distribution of nonradiative centres to be inferred.

Finally, we report recent progress in acquiring structural information using diffraction-based techniques in the EPMA. While the instrument geometry makes the integration of electron backscatter diffraction detectors difficult, we show that useful information on crystal deformation can be obtained using electron channelling contrast imaging (ECCI). This technique uses backscattered electrons to map subtle variations in the tilt and twist of the crystal, such as those between neighbouring grains or associated with the strain field around a single dislocation [2]. The ability to acquire such structural information concurrently with composition is key, as the two are often correlated; for example, features such as step bunches are often associated with preferential adsorption of one atomic species during growth [3]. 
Figure 1 shows a channelling image acquired from a sample of semi-polar AlGaN grown on a patterned substrate. Ignoring the vertical (topographic) features, this shows a number of extended defects including clear stacking faults (horizontal arrows) and possible threading dislocations (vertical arrows). The $310 \mathrm{~nm}$ monochromatic CL image (corresponding to the near band edge emission) exhibits contrast largely uncorrelated with the surface morphology, but shows reduced emission intensity in areas with higher defect densities. Figure 2 shows a further example, in this case of a c-plane (0001) AlGaN layer. The BSE image shows contrast not visible in secondary electron topographic images, including grain-tograin and intra-grain variations; these are only partly explained by atomic number contrast caused by Ga-rich areas at grain perimeters (seen also in the WDX and CL maps), with channelling accounting for the smaller scale details. This demonstrates the value of simultaneously acquiring multiple signal modes in samples where the optical, structural and compositional properties are so closely correlated [4].

\section{References:}

[1] M. J. Wallace et al, Journal of Applied Physics 116 (2014) p. 033105.

[2] G. Naresh-Kumar et al, Materials Science in Semiconductor Processing 47 (2016) p. 44.

[3] G. Kusch et al, Applied Physics Letters 104 (2014) p. 092114.

[4] The authors thank Prof. P. Parbrook of the Tydall National Institute and Prof. T. Wang of the University of Sheffield for providing samples. Funding is acknowledged from the UK EPSRC through grants EP/M015181/1, EP/P02744X/1 and EP/P015719/1.
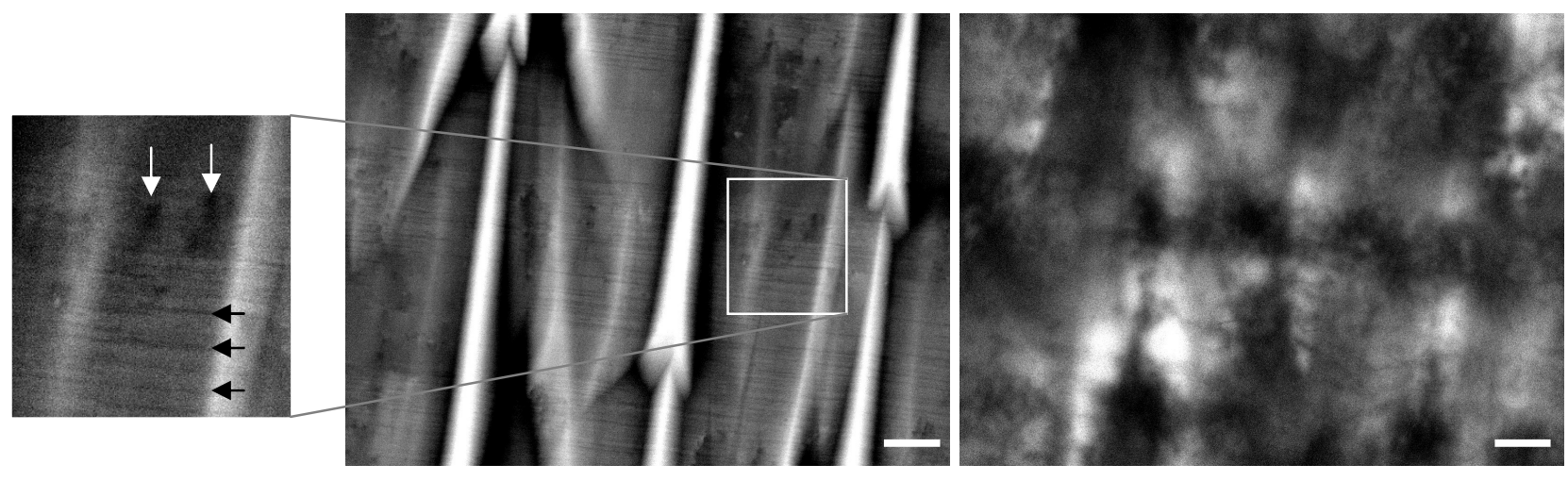

Figure 1. (Left and insert) backscattered electron and (right) $310 \mathrm{~nm} \mathrm{CL}$ image of $\mathrm{Al}_{0.38} \mathrm{Ga}_{0.62} \mathrm{~N}$ grown on a semi-polar plane using a patterned template. Acquired simultaneously at $15 \mathrm{kV}$; scale bar $1 \mu \mathrm{m}$.

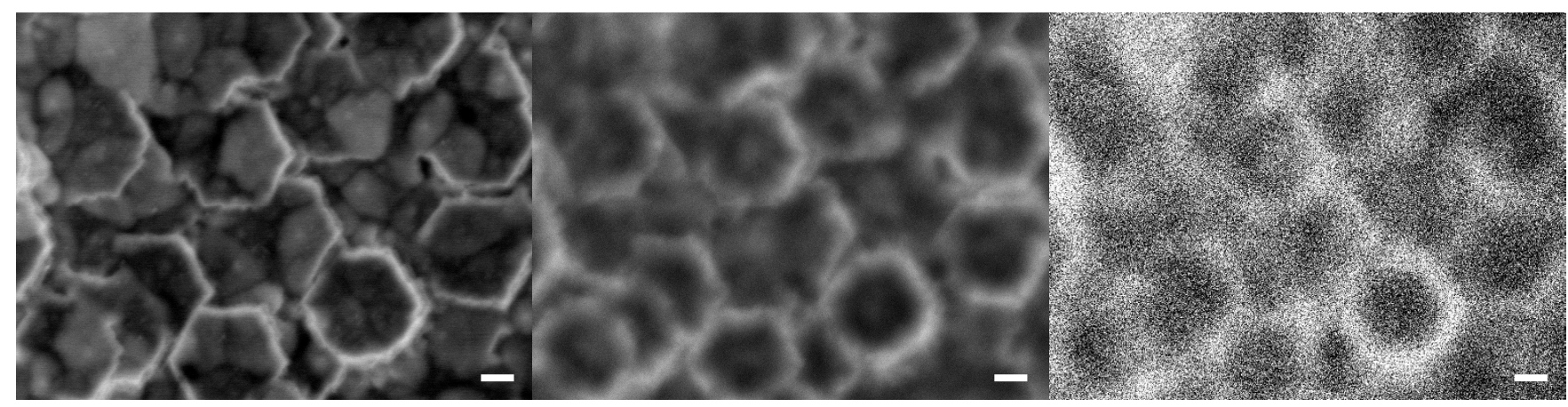

Figure 2. (Left) BSE image, (centre) panchromatic CL image and (right) uncalibrated WDX Ga $L_{\alpha}$ map of a $c$-plane $\mathrm{Al}_{0.82} \mathrm{Ga}_{0.18} \mathrm{~N}$ layer. Acquired at $10 \mathrm{kV}$; scale bar $1 \mu \mathrm{m}$. 